\title{
Combinational immunotherapy based on immune checkpoints inhibitors in small cell lung cancer: is this the beginning to reverse the refractory situation?
}

\author{
Haoyue Guo ${ }^{1,2}$, Yayi He ${ }^{1}$, Peixin Chen ${ }^{1,2}$, Lei Wang ${ }^{1}$, Wei Li $^{1}$, Bin Chen ${ }^{1}$, Yu Liu ${ }^{1,2}$, Hao Wang ${ }^{1,2}$, \\ Sha Zhao ${ }^{1,2}$, Caicun Zhou ${ }^{1}$ \\ ${ }^{1}$ Department of Medical Oncology, Shanghai Pulmonary Hospital, Tongji University Medical School Cancer Institute, Tongji University School of \\ Medicine, Shanghai, China; ${ }^{2}$ Tongji University, Shanghai, China \\ Contributions: (I) Conception and design: H Guo, Y He; (II) Administrative support: C Zhou; (III) Provision of study materials or patients: H Guo, P \\ Chen, L Wang, W Li; (IV) Collection and assembly of data: H Guo, B Chen, Y Liu, H Wang; (V Data analysis and interpretation: H Guo, H Wang, \\ S Zhao; (VI) Manuscript writing: All authors; (VII) Final approval of manuscript: All authors. \\ Correspondence to: Yayi He or Caicun Zhou. Department of Medical Oncology, Shanghai Pulmonary Hospital, Tongji University Medical School \\ Cancer Institute, Tongji University School of Medicine, No. 507 Zhengmin Road, Shanghai 200433, China. \\ Email: 2250601@qq.com or caicunzhoudr@126.com.
}

\begin{abstract}
Small cell lung cancer (SCLC), a particular neuroendocrine tumor, occupies $13 \%$ of lung cancers, with the highest mortality among cancers. Immune checkpoints inhibitors (ICIs) based on programmed cell death protein-1 (PD-1)/programmed cell death one ligand (PD-L1) inhibitors and cytotoxic T-lymphocyte-associated protein 4 (CTLA-4) inhibitors have been one of the most favorable therapies in SCLC. Simultaneously, not all the patients respond to ICIs due to the lack of biomarkers to predict the immunotherapeutic effect. Multiple combinational approaches are under exploration, including the integrated or successive assessment of additional immunotherapeutic agents, chemotherapy, radiotherapy, and targeted therapy with ICIs. The current review offers a general view of the rationale for clinical studies exploring the experimental result of combinational immunotherapy based on ICIs, with both available results and ongoing trials. Moreover, the development of more predictive biomarkers, specific clinical trial designs, enhancement of the efficacy, and decreasing the financial toxicity will become the trend of future research and clinical applications of ICIs. Understanding the evolving immuno-oncology is increasingly relevant and crucial to solve those problems and define therapeutic strategies and potential target populations of combinational immunotherapy. Ultimately, emerging combinational immunotherapy will transform SCLC into a chronic disease to help patients survive from tumors.
\end{abstract}

Keywords: Immune checkpoint inhibitors (ICIs); immunotherapy; small cell lung cancer (SCLC); targeted therapy; chemotherapy; radiotherapy

Submitted Apr 16, 2020. Accepted for publication Aug 21, 2020.

doi: $10.21037 /$ jtd-20-1689

View this article at: http://dx.doi.org/ 10.21037/jtd-20-1689

\section{Introduction}

Small cell lung cancer (SCLC), a particular neuroendocrine tumor, occupies $13 \%$ of lung cancers, with the highest mortality among cancers (1). SCLC consists of a limited stage (LS) and an extensive stage (ES). Although surgical resections are the most common therapy in cancers, SCLC, regardless of ES-SCLC or LS-SCLC, is usually treated with a combination of radiotherapy and chemotherapy, where the response is initially good but quickly developing resistance and relapse (1). Furthermore, the 5-year overall survival (OS) of ES-SCLC is merely $2 \%$ (2). Nowadays, immune checkpoint inhibitors (ICIs) are revolutionary representatives 
of immunotherapy to reverse refractory cancers, including SCLC, which is targeting the immune checkpoint pathway that tumor cells evade immune surveillance and progress.

However, immunotherapy based on ICIs faces a range of challenges in SCLC, including long response time and severe side effects. For example, the average response time of pembrolizumab in SCLC is 8.6 weeks (7.7-16.1 weeks), which is detrimental to some patients with high-grade malignant and rapidly progressing SCLC (3). Meanwhile, SCLC patients receiving immunotherapy are experiencing various degrees of side effects during the trial, even death (3). As a result, other immunotherapeutic agents, chemotherapy, radiotherapy, and targeted therapy are being developed to improve these defects. It has been validated that ICIs can be safely combined with a variety of immunotherapeutic agents, albeit at about half a dose.

Moreover, response rates were significantly higher with combinations of ICIs or other immunotherapy than monotherapy (4). Previous studies have found chemotherapy and radiation therapy can accelerate the development of tumor antigen-presenting cells (APCs), enhance the level of PD-L1 on tumor cells, and down-regulate pro-tumor immune cells at the tumor microenvironment (TME), such as regulatory $\mathrm{T}$ cells (Tregs) and myeloid-derivative suppressive cells (MDSC), and recruit $\mathrm{CD}^{+} \mathrm{T}$ cells and macrophages into the TME (5). The above evidence reveals the potential of chemotherapy and radiotherapy in enhancing the function of ICIs. Further, immunotherapy, combined with targeted therapy in cancer, can complement each other. Immunotherapy enhances the immune system's activity, reduces tumor tolerance to targeted therapy, and expands the beneficiary group of targeted therapy. On the other hand, the utilization of targeted therapy shortens the host response time to immunotherapy and activates the immune system through specific signal pathways.

The current review offers a general view of the rationale for clinical studies exploring the experimental result of combinational immunotherapy based on ICIs, with both available results and ongoing trials.

\section{The focus of immunotherapy: immune checkpoints and checkpoint inhibitors}

Cytotoxic T-lymphocyte-associated protein 4 (CTLA-4) and its inbibitors

CTLA-4 pathway in tumor immunity

CTLA-4 (CD152) is a surface or intracellular molecule on activated $T$ cells that curbs the initial period of $T$ cells activation as a competitive ligand for the T-cell costimulatory receptor CD28 (6). CTLA-4 is mostly found in intracellular compartments and is transported to the surface upon activated by $\mathrm{T}$ cell receptor (TCR) (7). Additionally, CTLA- 4 can both regulate $\mathrm{CD} 4^{+} \mathrm{T}$ cells and selective depletion of Tregs by removing CD80 and CD86 from the cell surfaces of antigen-presenting cells (APCs) via trans-endocytosis (8-10) (Figure 1). Further, a recent study developed a dual variable domain immunoglobulin of antiCTLA4 antibody, which could deplete intratumor Tregs, but spares tissue-resident Tregs, minimizing potential toxicities of CTLA-4 blockades (11).

\section{The efficacy of monotherapy with inhibitors of CTLA- 4 in SCLC}

There are two critical inhibitors of CTLA-4 currently studied in the treatment of lung cancers, which are tremelimumab (ticilimumab, CP-675,206) and ipilimumab (MDX-010, Yervoy, BMS-734016), hampering the joint of CTLA-4 on T cells with ligands (CD80/CD86) on APCs to enhance antitumor immune responses. Few completed clinical trials of ipilimumab or tremelimumab adopted monotherapy in SCLC patients. Therefore, combining inhibitors of CTLA-4 with additional therapies may lead to breakthroughs in SCLC treatment activity.

\section{The programmed cell death protein -1 (PD-1)/ programmed cell death one ligand (PD-L1) patbway and their inbibitors}

\section{PD-1/PD-L1 pathway in tumor immunity}

PD-1 (CD279) is mostly detected on the exterior of lymphocytes, primarily $\mathrm{CD}^{+} \mathrm{T}$ cells, and overexpressed on exhausted $\mathrm{CD}^{+} \mathrm{T}$ cells (Tex) (12). Currently, the ligands of PD-1 were confirmed to be PD-L1 (B7-H1) and PD-L2 (B7-DC), while PD-L1 is highly associated with tumor immunity. Besides tumor cells, PD-L1 and PD-L2 can also be found on other cells such as macrophages (13), myeloid DCs (14), MDSC (15), stromal fibroblasts (16), and endothelial cells (17). The expression of PD-1 not only inhibits $\mathrm{T}$ cells $\left(\mathrm{CD}^{+}\right)$mediated cell killing and promotes the differentiation of exhausted $\mathrm{CD}^{+} \mathrm{T}$ cells (Tex) (18) but also facilitates the differentiation of the Treg (CD4 Foxp $3^{+}$) cells from naive $\mathrm{CD}^{+} \mathrm{T}$ cells $(19,20)$. Moreover, the inhibitory effect of PD-1 on B cells improves immune responses to antigen-specific antibody, showing that $\mathrm{PD}-1$ can also impede B cell-mediated T-cell activation (20). 


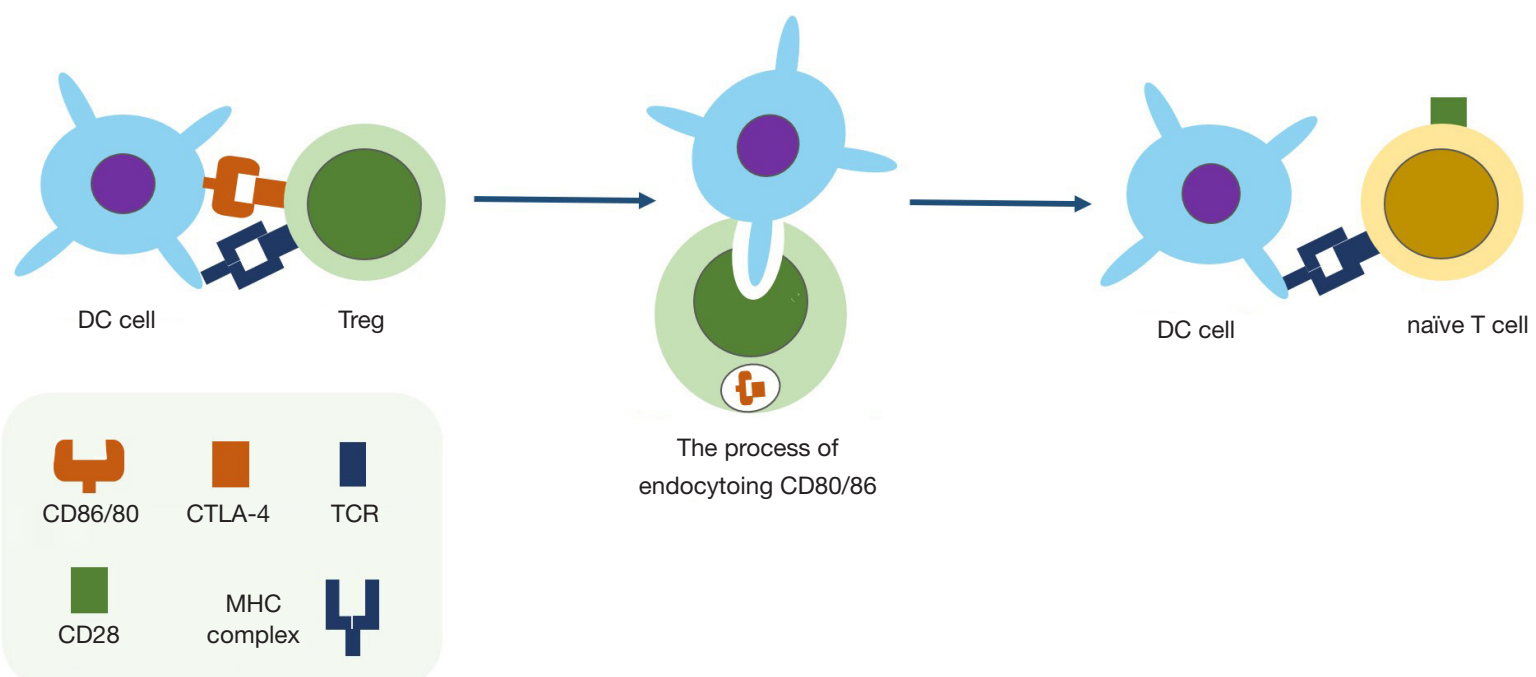

Figure 1 The immune suppression of Tregs via trans-endocytosis of CD80/86 from APCs. Upon activation by the combination of CTLA4: CD80/86, Tregs will endocytose CD80/86 on the surface of antigen presentation cells (APC), the binding receptor of the costimulatory receptor CD28 on naïve T cells. Therefore, the activated pathway of naïve T cells is blocked.

Importantly, the cytokines secreted by inflammatory cells, especially interferon- $\gamma$ (IFN- $\gamma$ ), can induce or maintain the PD-L1 protein expressions (21), showing an IFN- $\gamma /$ PD-L1 axis between tumor cells and the TME (Figure 2).

\section{The efficacy of monotherapy with inhibitors of PD-1/ PD-L1 in SCLC}

The inhibitors of the PD-1/PD-L1 that have been utilized in lung cancers are classified as anti-PD-1 agents and anti-PD-L1 agents. Nivolumab (opdivo, BMS 936558), durvalumab (imfinzi, MEDI4736), and pembrolizumab (Keytruda, MK-3475) are human-derived PD-1 monoclonal IgG4 antibodies in SCLC, which pair the PD-1 proteins expressed on $\mathrm{T}$ cells. Nivolumab monotherapy is approved in metastatic SCLC based on CheckMate 032, a significant study of nivolumab with or without ipilimumab (NCT01928394, phase I/II) (22,23). Durvalumab monotherapy was proved with enduring efficacy in some patients with the extensive pretreated disease (ED)-SCLC without additional security warnings (24). However, pembrolizumab monotherapy did not extend median progression-free survival (PFS) in ES-SCLC patients after receiving platinum and etoposide (25).

PD-L1 inhibitors also reported activity in the treatment of SCLC. Atezolizumab (tecentriq, MPDL3280A) is the earliest PD-L1 inhibitor in SCLC (26). Another clinical study investigating the outcomes and safety of atezolizumab in NSCLC or SCLC patients treated in routine clinical practice is ongoing (NCT03782207).

\section{Other immune checkpoints and their inhibitors}

In addition to the above two earliest and widely studied immune checkpoints, a few novel immune checkpoints and inhibitors such as $\mathrm{T}$ cell Ig and mucin-domain-3-containing molecule 3 (TIM-3), lymphocyte activation gene 3 (LAG-3) and et, al. have emerged in SCLC (Table 1).

Tim-3 is an immune checkpoint on $\mathrm{T}$ cells, FoxP3 ${ }^{+}$ Tregs, macrophages, DCs, nature killer (NK) cells, and mast cells to regulate immunity, upon interaction with ligands which are galectin-9 (Gal-9), carcinoembryonic antigen cell adhesion molecule 1 (CEACAM-1), high-mobility group protein B1 (HMGB1), and phosphatidylserine (PS) $(27,28)$. As a result, TIM-3 can hinder immune responses by inducing $\mathrm{T}$ cells exhaustion (29) and suppressing innate antitumor immunity, such as decreasing the production of IL-12 by monocytes (30). The expressions of TIM-3 on Tregs and NK cells were associated with lung cancer progression (31,32). Additionally, blocking TIM3 promotes the cytotoxicity of NK cells might be a promising target for immunotherapy in lung cancer (33).

LAG-3 (CD223) is an immune checkpoint that inhibits T cells stimulation and restrains cytokine production, therefore making the status of immune homeostasis (34). What is more, LAG-3 inhibits macrophages' differentiation or fully 


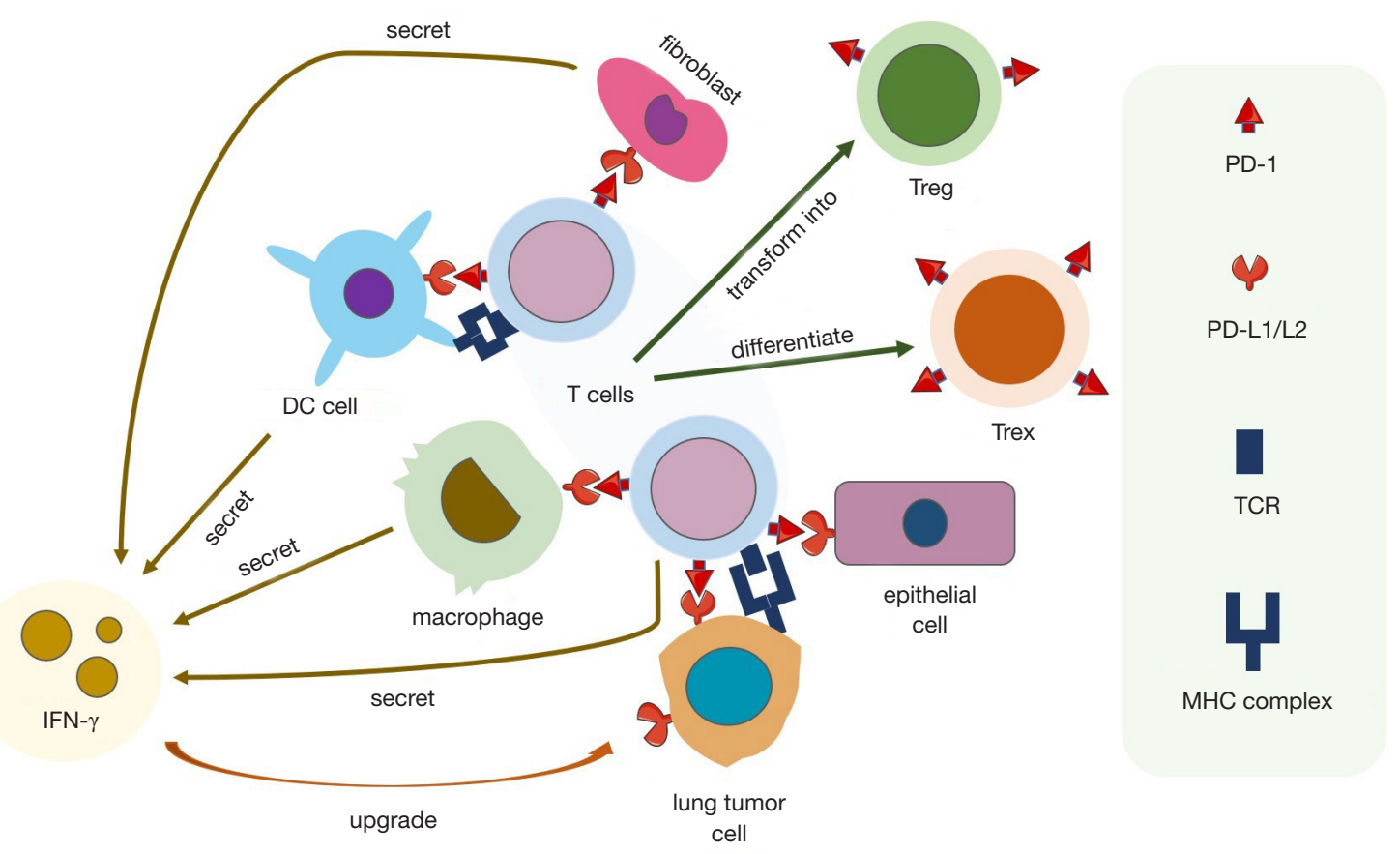

Figure 2 The suppression of antitumor immunity activated by the PD-1/PD-L1 pathway in the tumor microenvironment (TME) of lung cancers. The core of this network is tumor-specific T cells. The PD-1 protein is expressed both on activated T cells by the combination of major histocompatibility (MHC) complex with T cell receptor (TCR), and also exhausted CD8 ${ }^{+} \mathrm{T}$ cells (Tex). PD-L1 is expressed on various immune cells and stromal cells in TME. The engagement of the PD-1/PD-L1 pathway facilitates the transformation of Texs and the differentiation of regulatory T cells (Treg). Meanwhile, the secretion of IFN- $\gamma$ by infiltrating immune cells mentioned in the figure upgrades the expression of the PD-L1 protein.

competent antigen-presenting DCs from monocytes (35). Even though the exact process of LAG-3 signaling and interplay with additional immune checkpoints are generally unknown, LAG-3 blockades combined with PD-1 blockades are promising to suppress immune escape (34).

More immune checkpoints, along with their inhibitors involved in this review, were detailed in Table 1.

\section{Combinations with other immunotherapies}

\section{Combinations of immune checkpoints inbibitors}

The combination of ICIs is significant in SCLC treatment, most of which are the mixture of PD-1/PD-L1 and CTLA-4 pathway blockades, based on previous studies showing two pathways are nonredundant without additive or synergistic effects (36). Currently, there are two classic combos in the combination of PD-1/PD-L1 and CTLA-4 inhibitors, such as nivolumab plus ipilimumab and durvalumab plus tremelimumab.

Nivolumab \pm ipilimumab has been evaluated in advanced or metastatic SCLC patients after platinum therapy in phase I/II trial (CheckMate 032) (37). According to CheckMate032, both overall response rate and the incidence of grade 3-4 adverse effects (AEs) in nivolumab combined with ipilimumab (overall response rate: $23 \%$, AEs incidence: $33 \%)$ are higher than those in nivolumab monotherapy (overall response rate: $10 \%$, AEs incidence: 14\%), demonstrating a considerable tumor response and acceptable side effects in recurrent SCLC, regardless of PDL-1 expression and platinum-sensitivity/resistance. The same intervention was performed in another randomized expansion cohort, with an overall response rate of $12 \%$ and $21 \%$ in nivolumab monotherapy and nivolumab plus ipilimumab, sharing a similar 3-month OS of $64 \%$ and $65 \%$ in monotherapy and combined therapy (23). Based on these promising results, more clinical trials on nivolumab combined with ipilimumab are ongoing (Table 2). A phase III trial (CheckMate 451; NCT02538666) is ongoing to evaluate nivolumab plus ipilimumab compared with nivolumab monotherapy and placebo for ES-SCLC 
Table 1 Selected immune checkpoint inhibitors in SCLC

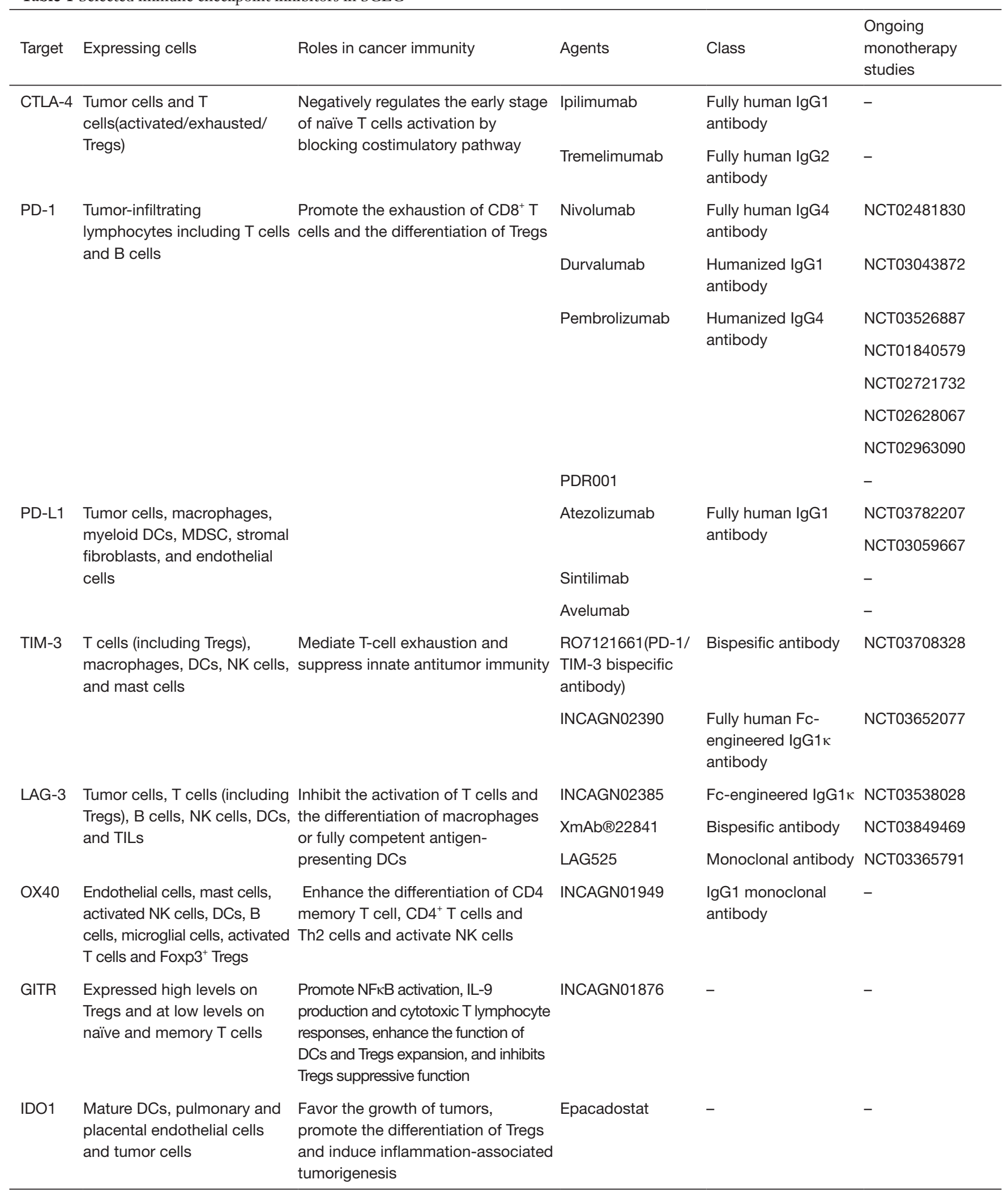

Table 1 (continued) 
Table 1 (continued)

\begin{tabular}{|c|c|c|c|c|c|}
\hline Target & Expressing cells & Roles in cancer immunity & Agents & Class & $\begin{array}{l}\text { Ongoing } \\
\text { monotherapy } \\
\text { studies }\end{array}$ \\
\hline CD137 & $\begin{array}{l}\text { Tumor cells, } \mathrm{CD}^{+} \text {and } \mathrm{CD} 8^{+} \mathrm{T} \\
\text { cells, Foxp } 3^{+} \text {Tregs, NK cells, } \\
\text { and DCs }\end{array}$ & $\begin{array}{l}\text { Activate and proliferate monocytes } \\
\text { and NK cells, promote T cell activation, } \\
\text { proliferation, and differentiation, and } \\
\text { also induce } T \text { cell apoptosis }\end{array}$ & Utomilumab & $\begin{array}{l}\text { IgG2 monoclonal } \\
\text { antibody }\end{array}$ & - \\
\hline
\end{tabular}

CTLA-4, cytotoxic T-lymphocyte-associated protein 4; PD-1, programmed cell death protein-1; PD-L1, programmed cell death 1 ligand; TIM-3, T cell Ig and mucin-domain-3-containing molecule 3; LAG-3, lymphocyte-activation gene 3; GITR, glucocorticoid-induced TNFRrelated protein; IDO1, indoleamine 2, 3-dioxygenase 1; TIGT, tyrosine-based inhibitory motif domain; Tregs, regulatory T cells; NK cells, nature killer cells; TILs, tumor infiltrating lymphocytes; MDSC, myeloid suppressor cells.

after first-line platinum-based doublet chemotherapy (PT-DC) (38). Moreover, NCT03670056 observes the changes in tumor antigen-specific effector $\mathrm{T}$ (Teff)/ Treg cells as a primary outcome measure, which strongly influences antitumor immunity (23). Further, triple ICIs combinations based on nivolumab plus ipilimumab are also started to come out. A phase I/II trial explores the security, tolerability, and efficacy of INCAGN01876, an anti-GITR agonistic monoclonal antibody, plus nivolumab and (or) ipilimumab in advanced or metastatic malignancies including SCLC. Similarly, another attempt to combine an anti-OX40 agonistic antibody INCAGN01949 with nivolumab and ipilimumab in advanced or metastatic malignancies has been recently completed, but the result has not been reported (NCT03241173; phase I/II).

The combination of durvalumab plus tremelimumab has been confirmed to show antitumor activity in NSCLC, regardless of $\mathrm{PD}-\mathrm{L} 1$ status, with an objective response rate of $23 \%$ (36). As a result, similar clinical trials are being conducted in SCLC to investigate whether durvalumab plus tremelimumab has a more potent antitumor activity than either drug alone (Table 2). The ADRIATIC (NCT03703297) is a phase III study in durvalumab with or without tremelimumab for patients with LS-SCLC following chemoradiation therapy to compare PFS and OS (approximately 5 years). An ongoing phase II study in ES-SCLC compares the combination of durvalumab plus tremelimumab with another two chemotherapies, based on overall response rate and other indexes (NCT02937818). The rest ongoing studies attempt to build a joint therapy of durvalumab plus tremelimumab, with chemotherapy or radiotherapy (NCT03923270, NCT02701400,

\section{NCT03043872, NCT03509012).}

Except for these two classic combos, there are more new combinational ICIs combos. The first star agent is pembrolizumab, which is explored to be combined with ipilimumab (NCT01840579), XmAb®22841 (a CTLA-4 x LAG-3 bispecific antibody; NCT03849469), INCAGN01876 (a GITR agonist antibody; NCT03277352) and epacadostat (an inhibitor of indoleamine 2,3-dioxygenase-1 (IDO1); NCT03277352, NCT03361228), which are still at initial stages. A phase III study named SKYSCRAPER-02 exerts an efficacy comparison between tiragolumab plus atezolizumab, cisplatin (or carboplatin), and etoposide (EC) with placebo plus atezolizumab and EC (NCT04256421). Finally, the JAVELIN Medley (NCT02554812) explores the safety and effect of avelumab plus utomilumab in advanced malignancies, including SCLC. Another ongoing phase II trial (NCT02554812) determines whether a PD-1 blockade PDR001 plus a LAG-3 inhibitor LAG525 demonstrates sufficient efficacy in advanced malignancies, including SCLC.

\section{Combinations with other immunotherapeutic agents}

\section{Agonists of cytokines and proteins}

Interleukin (IL-15) is a promising target in immunotherapy, which can upgrade the production, function, and survival of $\mathrm{CD}^{+} \mathrm{T}$ and NK cells (39). A current report has shown that the combination of IL-15 superagonist named ALT803 and nivolumab in metastatic NSCLC was well-tolerated, with an objective response rate of $29 \%$ (40). Meanwhile, the addition of AL-803 may increase the objective responses in 
Table 2 Ongoing studies on combination of ICIs and other immunotherapeutic agents in SCLC

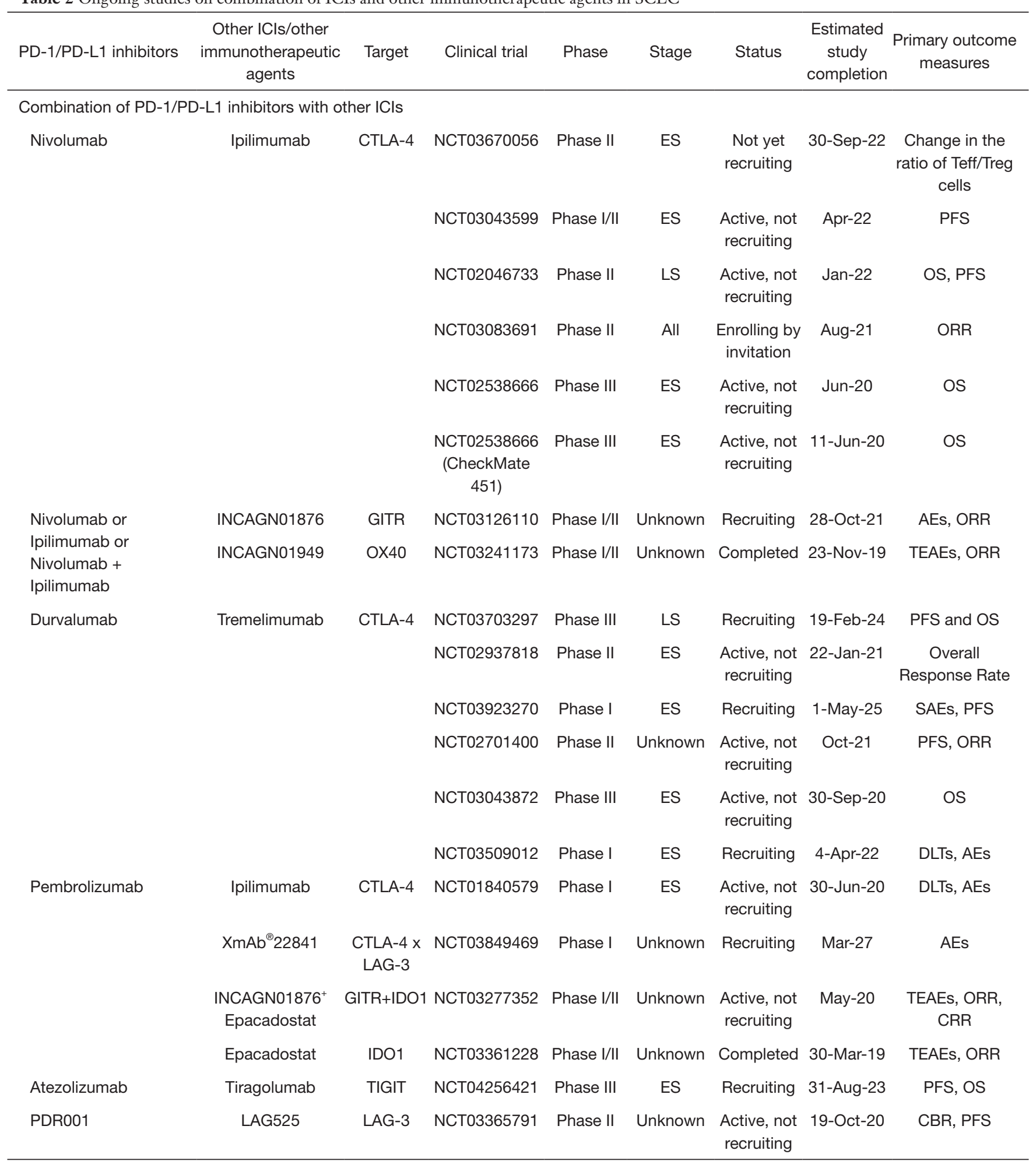

Table 2 (continued) 
Table 2 (continued)

\begin{tabular}{|c|c|c|c|c|c|c|c|c|}
\hline PD-1/PD-L1 inhibitors & $\begin{array}{c}\text { Other ICls/other } \\
\text { immunotherapeutic } \\
\text { agents }\end{array}$ & Target & Clinical trial & Phase & Stage & Status & $\begin{array}{l}\text { Estimated } \\
\text { study } \\
\text { completion }\end{array}$ & $\begin{array}{l}\text { Primary outcome } \\
\text { measures }\end{array}$ \\
\hline $\begin{array}{l}\text { Nivolumab or } \\
\text { Pembrolizumab or } \\
\text { Atezolizumab or } \\
\text { Avelumab }\end{array}$ & ALT-803 & IL-15 & NCT03228667 & Phase II & Unknown & Recruiting & Aug-20 & ORR \\
\hline Pembrolizumab & SO-C101 & IL-15 & NCT04234113 & Phase I & Unknown & Recruiting & Mar-22 & $\begin{array}{c}\text { DLTs, AEs, SAEs, } \\
\text { laboratory test } \\
\text { abnormalities, } \\
\text { Clinically } \\
\text { Significant } \\
\text { Change and } \\
\text { ECOG }\end{array}$ \\
\hline Atezolizumab & BNT411 & TLR7 & NCT04101357 & Phase I/II & ES & $\begin{array}{l}\text { Not yet } \\
\text { recruiting }\end{array}$ & Dec-23 & $\begin{array}{l}\text { DLTs, TEAEs, } \\
\text { IMP dose } \\
\text { reductions, } \\
\text { IMP treatment } \\
\text { discontinuations } \\
\text { due to toxicity }\end{array}$ \\
\hline Nivolumab & BMS-986012 & $\begin{array}{c}\text { fucosyl- } \\
\text { GM1 }\end{array}$ & NCT02247349 & Phase I/II & Unknown & $\begin{array}{l}\text { Active, not } \\
\text { recruiting }\end{array}$ & 28-Sep-20 & $\begin{array}{c}\text { AEs, worst grade } \\
\text { SAEs }\end{array}$ \\
\hline $\begin{array}{l}\text { Nivolumab + } \\
\text { Ipilimumab }\end{array}$ & $\begin{array}{c}\text { INGN-225 (Ad. p53- } \\
\text { DC) }\end{array}$ & $\begin{array}{l}\text { Dendritic } \\
\text { cell p53 }\end{array}$ & NCT03406715 & Phase II & $\mathrm{LS}+\mathrm{ES}$ & Recruiting & Apr-21 & DCR \\
\hline
\end{tabular}

ICls, immune checkpoint inhibitors; OS, overall survival; PFS, progression-free survival; LS, limited stage; ES, extensive stage; AEs, adverse events; DCR, disease control rate; IMP, investigational medicinal products; DLTs, dose-limiting toxicities; SAEs, serious adverse events; MTD, maximum tolerated dose; ORR, objective response rate; RR, response rate; CRR, complete response rate; TEAEs, treatment-emergent adverse events; CBR, clinical benefit rate; Cmax, maximum plasma concentration; ADA, anti-drug antibody; TTR, time to tumor response; DR, duration of response; ECOG, Eastern Cooperative Oncology Group.

patients with PD-1-resistance (40). Based on this research, more trials are ongoing for applying IL-15 superagonists to assist PD-1/PD-L1 blockades in SCLC. NCT03228667 (phase II) sets up four interventions, including ALT-803 plus pembrolizumab or nivolumab or atezolizumab or avelumab in SCLC patients. Further, NCT04234113 looks into whether there is an improvement or decreased safety in SO-C101 combined with pembrolizumab, another IL-15 superagonist, compared with SO-C101 alone.

Toll-like receptor-7 (TLR-7) has been detected on the endosomes of immune cells such as macrophages, NK cells, DCs, and B cells. Activation of TLR7 ligands is beneficial 
to the differentiation and stimulation of immune cells and the secretion of pro-inflammatory cytokines (41). A TLR-7 agonist called BNT411 can amplify the potential immunestimulating and antitumor activities of TLR-7, combined with atezolizumab and EC in an ongoing phase I/II trial (NCT04101357) in ES-SCLC.

\section{Agents targeting tumor surface proteins}

The ganglioside fucosyl-GM1 (FucGM1) is a specific tumor antigen on SCLC cells, but missing in normal tissues, which is a favorable therapeutic option for specific antitumor immunity. Previous researches had shown that a fully human anti-fucosyl-GM1 antibody (BMS-986012) may induce antitumor activity by complement-dependent cytotoxicity (CDC) and antibody-dependent cellular phagocytosis (ADCP) assays, while the tumor response enhanced when BMS-986012 was in combination with PD-1 or CD137 blockades (42). Therefore, a phase I/II study (NCT02247349) is ongoing to detect the effect and safety of the combination of BMS-986012 and nivolumab in relapsed or refractory SCLC.

The Wilms' tumor suppressor gene (WT1) was initially recognized as a tumor suppressor gene in Wilm's tumor and was then found overexpression in various solid tumors, including lung cancer (43). Caihua $\mathrm{Xu}$ et al. found that WT1 promoted proliferation of NSCLC cells by upgrading Cyclin D1 and p-pRb (44). Galinpepimut-S (GPS) is a novel cancer vaccine that targets the WT1 protein and has been adopted by FDA for mesothelioma. Hence, a phase I/II study is recruiting to combine pembrolizumab with GPS in selected advanced cancers, including SCLC.

\section{Agents targeting innate immune cells}

FT500 is a first NK cell immunotherapeutic agent produced from a self-renewing clonal master pluripotent cell line (MPCL). Current studies have found that FT500 can facilitate the recruitment and activation of tumorinfiltrating lymphocytes (TILs), suggesting an ability to reduce tumor burden and resistance of ICIs (45). A phase I study on FT500 monotherapy and in combination of nivolumab, pembrolizumab, and atezolizumab along with chemotherapy in selected advanced solid tumors, including SCLC, is recruiting.

p53 gene mutations often occur in cancers, leading to tumor proteins' expression and making p53-specific cytotoxic $\mathrm{T}$ cells recognize tumor cells. Thence, INGN225 (Ad. p53-DC), a p53-modified adenovirus-transduced dendritic cell vaccine, was developed, which has been proved well tolerated in SCLC, with all side effects $\leq$ grade 2 in a phase I/II trial (NCT03406715) (46). Also, specific anti-p53 immune responses and overall post-INGN-225 responses were observed in $41.8 \%$ and $51.5 \%$ of SCLC patients, respectively (46). Considering these postINGN-225 immune responses, a phase II trial is ongoing to combine Ad. p53-DC with ipilimumab and nivolumab in LS-SCLC to explore whether there is an enhanced antitumor activity of ICIs (NCT03406715).

\section{Combinations with chemotherapy or(and) radiotherapy}

Chemotherapy and radiotherapy are beneficial to mature antigen-presenting cells, increase PD-L1 expression on tumor cells, decrease tumor-infiltrating Tregs or MDSCs, and recruit $\mathrm{CD} 8^{+}$TILs and macrophages (5). These shaping of TME from chemotherapy and radiotherapy make them become the first choice in combination with immunotherapy.

\section{Combinations with chemotherapy}

NCT01331525 proved the combination of ipilimumab with EC improved a subgroup of patients' outcomes but also caused severe neurological toxicity in advanced SCLC (47). $72.4 \%$ and $84.8 \%$ of patients presented an objective response by RECIST and the immune-related response criteria. However, grade 3 or higher toxicity occurred in $89.7 \%$ of patients. Moreover, another phase III study (NCT01450761) revealed that the addition of ipilimumab to etoposide and platinum did not improve OS compared with chemotherapy alone in ES-SCLC patients, also without new adverse events (48). Thus, current researches shift the redemptions to the combination of other ICIs with chemotherapy.

A phase II trial (NCT02551432) has shown a moderate activity with acceptable toxicity of pembrolizumab combined with paclitaxel in refractory ES-SCLC, where the objective response rate was $23.1 \%$, among which complete response rate is $3.8 \%$ and confirmed partial response is $19.2 \%$ (49). Various kinds of grade 3 or 4 AEs appeared among 4-8\% patients. However, a significantly decreased NK cell activity was found in responders from flow cytometric analysis. Moreover, the PembroPlus (NCT02331251) explored the possible combos of pembrolizumab combined with common chemotherapies, including gemcitabine (G), docetaxel (D), nab-paclitaxel (NP), vinorelbine (V), irinotecan (I) and 
liposomal doxorubicin (LD) in advanced cancers including SCLC. The result showed the safety of standard-dose pembrolizumab combination with $\mathrm{G}, \mathrm{G}+\mathrm{NP}, \mathrm{G}+\mathrm{V}$, I, and $\mathrm{LD}$, indicating possible future research directions (50). Recently, MK-3475-604/KEYNOTE-604 (NCT03066778; phase III) has shown mixed results in ES-SCLC that pembrolizumab plus etoposide/platinum could potentially prolong PFS ( $\mathrm{P}=0.0023$; median 4.5 vs. 4.3 months) but OS ( $\mathrm{P}=0.0164$; median 10.8 vs. 9.7 months) (51). Further, pembrolizumab is being combined with more chemotherapies, such as topotecan (NCT02963090), amrubicin (NCT03253068), EC (NCT03043872, NCT02580994, NCT01840579), paclitaxel or pemetrexed (NCT01840579) (Table 3).

IMpower133 (NCT02763579) demonstrated a synergistic potential of ICIs combined with first-line chemotherapy, that the addition of atezolizumab to EC in ED-SCLC brought remarkably extended OS and PFS than chemotherapy alone, with a manageable safety profile (52). Based on this promising result, emerging clinical trials on the addition of atezolizumab to EC (NCT04028050, NCT02763579, NCT04221529) with placebo (NCT03041311) in ES-SCLC are ongoing. Moreover, the SKYSCRAPER-02 (NCT04256421) is probing whether atezolizumab plus EC combined with tiragolumab have a different efficacy from atezolizumab plus EC alone in ESSCLC.

Nivolumab, in combination with PT-DC has shown that nivolumab plus pemetrexed-cisplatin or paclitaxelcarboplatin or gemcitabine-cisplatin, achieved an objective response rate, a 24-week PFS and a 2-year OS up to $47 \%$, $71 \%$ and $62 \%$, respectively (NCT01454102) (53). Herein, clinical scientists are exploring the potential combos of nivolumab plus more chemotherapies, including irinotecan (NCT04173325), temozolomide (NCT03728361), gemcitabine (NCT03662074), and EC (NCT03382561) (Table 3).

An ongoing phase III trial (CASPIAN; NCT03043872) proved that durvalumab combined with platinum-etoposide was related to a considerably increased survival (median OS: 13 months, 18 -month OS: $34 \%$ ) from the platinumetoposide group (median OS: 10.3 months, 18-month OS: $25 \%$ ). Meanwhile, the incidence of grade 3 or $4 \mathrm{AEs}$ is the same between the two groups, at $62 \%$ (54). Another ongoing phase II trial (MEDIOLA) has revealed the combination of olaparib and durvalumab was relatively well tolerated, but the efficacy did not reach the target disease control rates (DCRs) and was below the futility boundary
$(<40 \%)$ (55). ADRIATIC (NCT03703297) is an ongoing phase III trial to study the combo of durvalumab and placebo, compared with durvalumab plus tremelimumab, and placebo alone in LS-SCLC.

Finally, PAVE (NCT03568097, phase II) is in the process to explore the mixture of avelumab with first-line chemotherapy of EC in SCLC patients with carcinoma.

\section{Combinations with radiotherapy}

177Lu-DOTATATE (lutathera) is a promising target in patients with metastatic thoracic neuroendocrine tumors that express somatostatin receptors (56). An ongoing phase I/II trial (NCT03325816) has shown early evidence that lutathera combined with nivolumab is safe and welltolerated with initial signs of antitumor activity (57). NCT02978404 (phase II) is looking into combining nivolumab and stereotactic radiosurgery (SRS), which is increasingly applied as the sole treatment of brain metastases in selected cancers, including SCLC. Stereotactic body radiation (SBRT) therapy (NCT03223155) is also being tested to combine with the classic combo of nivolumab plus ipilimumab in stage-IV SCLC.

An ongoing randomized phase II study of tremelimumab plus durvalumab \pm SBRT, showing no significant difference of efficacy for ICIs with or without SBRT in relapsed SCLC (58). Further, NCT03262454 (phase II) is attempting to add atezolizumab to the hypofractionated radiation therapy in SCLC.

\section{Combinations with chemoradiotherapy}

Most chemoradiotherapy combined with ICIs in current trials of SCLC are based on first-line EC, including durvalumab (NCT03585998, NCT03509012), pembrolizumab (NCT02934503, NCT02402920, NCT02402920) and atezolizumab (NCT03540420, NCT03811002) (Table 3). Of note, the CLOVER (NCT03509012, phase I) is setting four arms to evaluate the addition of tremelimumab to the combination of durvalumab and EC with external beam radiation, determining the different efficacy between hypofractionated and standard external beam radiation.

Except for the combination with EC, NCT03923270 (phase II) is setting three arms to assess the activity and safety of durvalumab plus thoracic radiotherapy, durvalumab combined tremelimumab plus thoracic radiotherapy and durvalumab combined olaparib pills plus thoracic 
Table 3 Ongoing studies on combination of ICIs and chemotherapy and (or) radiotherapy in SCLC

\begin{tabular}{|c|c|c|c|c|c|c|c|}
\hline $\mathrm{ICls}$ & $\begin{array}{c}\text { Chemotherapy/ } \\
\text { radiotherapy/chemo- } \\
\text { radiotherapy }\end{array}$ & Clinical trial & Phase & Stage & Status & $\begin{array}{l}\text { Estimated } \\
\text { study } \\
\text { completion }\end{array}$ & $\begin{array}{l}\text { Primary outcome } \\
\text { measures }\end{array}$ \\
\hline Nivolumab & Irinotecan & NCT04173325 & Phase I & Unknown & Recruiting & Nov-21 & AEs \\
\hline Nivolumab & $\mathrm{EC}$ & NCT03382561 & Phase II & ES & $\begin{array}{l}\text { Active, not } \\
\text { recruiting }\end{array}$ & 2-Jun-20 & PFS \\
\hline Nivolumab & Temozolomide & NCT03728361 & Phase II & Stage IV & Recruiting & 31-Dec-21 & ORR \\
\hline Nivolumab & Gemcitabine & NCT03662074 & Phase II & Stage IV & Recruiting & 10-Sep-21 & Radiographic RR \\
\hline Durvalumab & Placebo & NCT03703297 & Phase III & LS & Recruiting & 19-Feb-24 & PFS, OS \\
\hline Durvalumab & Olaparib & NCT02734004 & Phase I/II & Unknown & Recruiting & 5-Aug-22 & DCR, ORR \\
\hline Pembrolizumab & Topotecan & NCT02963090 & Phase II & Unknown & $\begin{array}{l}\text { Active, not } \\
\text { recruiting }\end{array}$ & 20-Aug-19 & PFS \\
\hline Pembrolizumab & Amrubicin & NCT03253068 & Phase II & ES & Recruiting & 31-Aug-20 & OS \\
\hline Pembrolizumab & EC & NCT03066778 & Phase III & ES & $\begin{array}{l}\text { Active, not } \\
\text { recruiting }\end{array}$ & $5-O c t-21$ & PFS, OS \\
\hline Pembrolizumab & EC & NCT02580994 & Phase II & ES & $\begin{array}{l}\text { Active, not } \\
\text { recruiting }\end{array}$ & Aug-20 & PFS \\
\hline Atezolizumab & EC + Placebo & NCT03041311 & Phase II & ES & $\begin{array}{l}\text { Active, not } \\
\text { recruiting }\end{array}$ & May-20 & $\begin{array}{l}\text { Chemotherapy- } \\
\text { induced } \\
\text { myelosuppression }\end{array}$ \\
\hline Atezolizumab & EC & NCT04028050 & Phase III & ES & Recruiting & 30-Dec-22 & $\begin{array}{c}\text { SAEs, Serious and } \\
\text { Non-Serious Immune } \\
\text { Related Adverse } \\
\text { Events }\end{array}$ \\
\hline Atezolizumab & EC & NCT02763579 & Phase III & ES & $\begin{array}{l}\text { Active, not } \\
\text { recruiting }\end{array}$ & 30-Dec-20 & PFS, OS \\
\hline Atezolizumab & EC & NCT04221529 & Phase II & ES & Recruiting & Jun-24 & OS \\
\hline Avelumab & $\mathrm{EC}$ & NCT03568097 & Phase II & Unknown & Recruiting & Aug-21 & 1-year PFS \\
\hline
\end{tabular}

Table 3 (continued) 
Table 3 (continued)

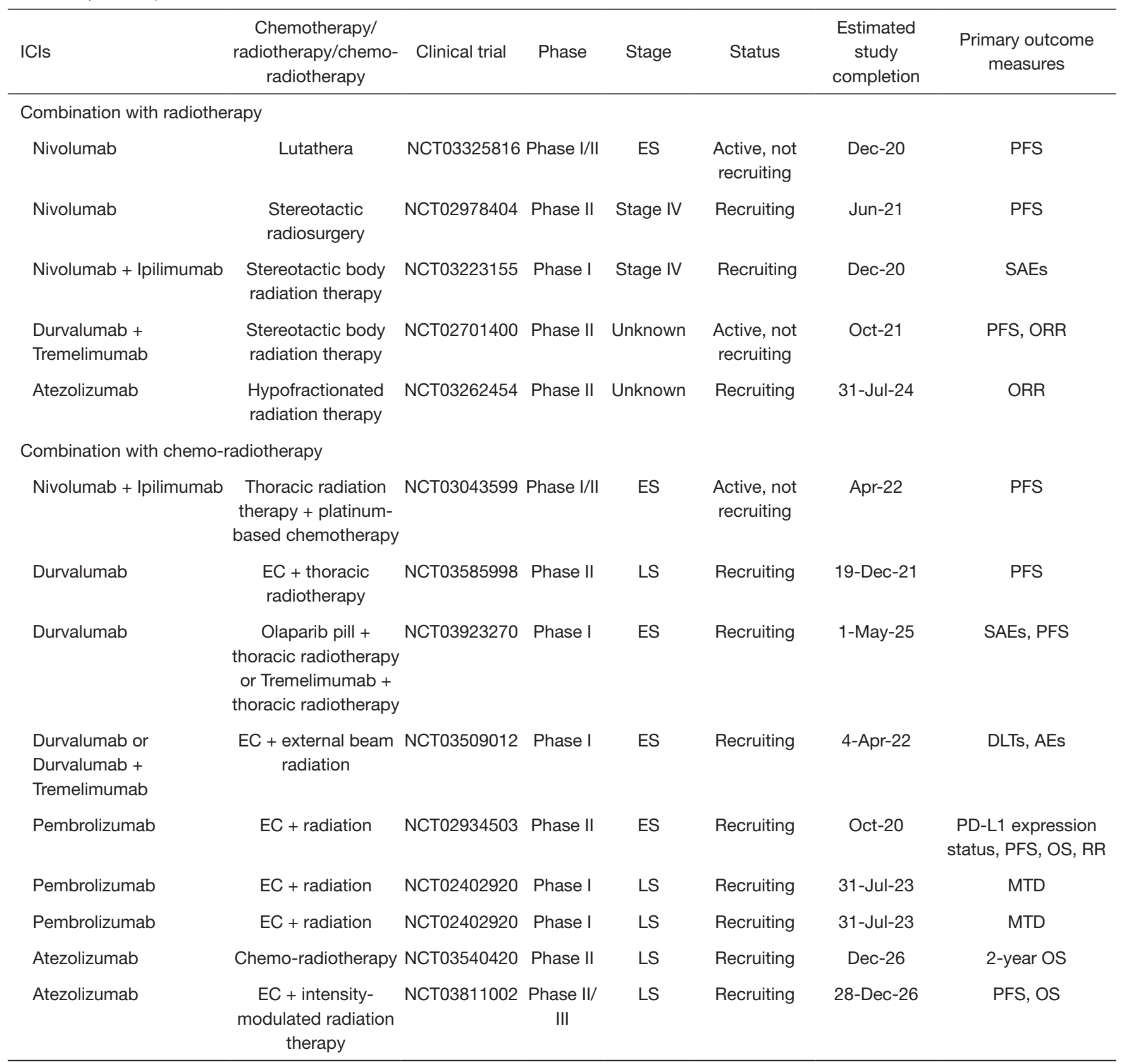

ICls, immune checkpoint inhibitors; OS, overall survival; PFS, progression-free survival; EC, cisplatin or carboplatin plus etoposide; LS, limited stage; ES, extensive stage; AEs, adverse events; DCR, disease control rate; DLTs, dose-limiting toxicities; SAEs, serious adverse events; MTD, maximum tolerated dose; ORR, objective response rate; $R R$, response rate.

radiotherapy.

Finally, a prospective single-arm phase I/II study (NCT03043599) utilized ipilimumab and nivolumab with consolidative thoracic radiotherapy after platinum doublet chemotherapy for patients with ES-SCLC. The result did not significantly improve the 6-month PFS compared to historical estimates, while the OS estimate at one year compares favorably with historical estimates (59). 


\section{Combinations with targeted therapy}

\section{Antiangiogenic agents}

A series of mice models demonstrated that the antiangiogenic therapy targeting vascular endothelial growth factor (VEGF) and Angiopoietin-2 (ANG2) (60), and vascular endothelial growth factor receptor 2 (VEGFR2) (61) raised the level of PD-L1 on endothelial cells (ECs) and tumor cells, leading to the suppression of antitumor immunity. Thus, a piece of equitable evidence supports the combination of antiangiogenic agents and ICIs, to solve the resistance during the antiangiogenic therapy.

\section{Tyrosine kinase inhibitors (TKIs)}

Receptor tyrosine kinases (RTKs) are transmembrane glycoproteins, which serve as the connection of cellular growth factors and extracellular ligands (62). Currently, targeted RTK inhibitors have been a new representative of targeted therapy in cancers, including SCLC (63).

Anlotinib (AL3818) is an orally multiple TKI, targeting c-kit, fibroblast growth factor receptor (FGFR), VEGFR, and platelet-derived growth factor receptor (PDGFR), which has been permitted for the treatment in NSCLC in China (62). Based on these outcomes in NSCLC, two clinical trials are recruiting to explore more potential of anlotinib combined with nivolumab (NCT04165330) and sintilimab (NCT04055792) in SCLC.

Vorolanib (X-82) is an orally dual inhibitor targeting VEGFRs and PDGFRs with antiangiogenic and antineoplastic activities, which has demonstrated reduced tissue toxicity by $95 \%$ with first-generation kinase inhibitors. NCT03583086 (phase I/II) estimates the tolerability and effect of nivolumab plus vorolanib in patients with refractory thoracic tumors, including SCLC.

Cabozantinib (XL184), an orally bioavailable TKI with activity against MET, VEGFR 2, AXL, ROS1, and RET, has been tested in some advanced solid tumors (64). NCT04079712 is a recruiting phase II trial to test the combination of XL184, nivolumab, and ipilimumab for differentiated neuroendocrine cancers.

\section{VEGF inhibitors}

VEGF is immensely expressed in SCLC cells, and it is related to cancer cell proliferation, metastasis, and invasion, while clinical evaluation of angiogenesis inhibitors has not improved OS in SCLC (2). Bevacizumab is a humanized anti-VEGF-A monoclonal $\mathrm{IgG}_{1}$ antibody (65), which is also the VEGF inhibitor most studied in SCLC (2).
NCT02734004 (phase I/II) is a study to assess the antitumor activity and safety of the mixture of bevacizumab, durvalumab, and olaparib, compared with the other arms of durvalumab plus olaparib, and bevacizumab plus olaparib.

\section{Cyclin-dependent protein kinase (Cdks) inbibitors}

Once combing with cyclin $\mathrm{D}$, cyclin-dependent kinases (CDK4/6) firstly phosphorylate retinoblastoma protein $(\mathrm{Rb})$, leading to the cell transition from $\mathrm{G} 1$ to $\mathrm{S}$ phase (66). CDK4/6 inhibitors can obstruct cell cycle and induce cell senescence and disruption by a cytotoxic T-cell-mediated effect in cancer (67). Trilaciclib is a CDK4/6 inhibitor that has been used for breast cancer in postmenopausal women. In SCLC, NCT03041311 (phase II) is in the process of evaluating the addition of trilaciclib to the combination of carboplatin, etoposide, and atezolizumab in ES-SCLC.

Additionally, a selective CDK7 inhibitor, YKL-5-124, was another potent treatment in SCLC mice models in Hua Zhang et al.'s study (68). Anti-PD-1 + YKL-5-124 presented with a better response than a single treatment. Strikingly, the mice treated with four-drug combination (YKL-5-124 + anti-PD-1+EC) appeared to live significantly longer than those treated with anti-PD-1+EC or EC alone. Moreover, this study also indicated that YKL-5-124 induced antitumor immune reactions engaging $\mathrm{DCs}\left(\mathrm{MHCII}^{+} \mathrm{CD} 11 \mathrm{c}^{+} \mathrm{CD} 103^{+}\right)$, effector $\mathrm{CD}^{+}{ }^{+} \mathrm{T}$ cells $\left(\mathrm{CD} 44^{\text {high }} \mathrm{CD} 62 \mathrm{~L}^{\text {low }} / \mathrm{Ki} 67^{+} / \mathrm{ICOS}^{+}\right)$ and cytotoxic CD8+T cells (Granzyme $\mathrm{B}^{+}$), which could be heightened by the combination with PD-1 blockades.

\section{Arginase and its inbibitor}

Arginase in the TME can inhibit antitumor NO secreted by pro-inflammation macrophages, synthesis of polyamines supporting tumor cells multiplication, and topical arginine reduction, which result in T-cell dysfunction and immunosuppression (69). Current studies have demonstrated that arginase was expressed on various cancers (69) while its inhibitors could cause suppression of breast cancer cell proliferation (70). INCB001158 is an orally available inhibitor of arginase, which inhibits the breakdown of arginine by arginase and restores arginine levels. Thus, NCT03361228 (phase I/II) is a study to explore the security, tolerability, and preclinical effect of INCB001158 plus epacadostat, with or without pembrolizumab in advanced solid tumors, including SCLC.

Arginine is a kind of semi-essential amino acid, while several cancers fail to endogenously synthesize enough 
arginine for survival and proliferation, mainly because of decreased level of argininosuccinate synthase (ASS1) (71). Pegzilarginase is a highly potent human arginine-degrading enzyme, while SCLC has been validated with the highest incidence of decreased ASS1 expression and the highest rate of responding to pegzilarginase in patient-derived xenografts (PDX) models (71). Moreover, pegzilarginase prevented tumor growth and promoted the infiltration of $\mathrm{CD}^{+} \mathrm{T}$ cells in the TME in mouse models (71). Thus, an ongoing phase I/II study (NCT03371979) explores the security and effect of pegzilarginase plus pembrolizumab in ED-SCLC with relapsed or progressive disease on or in 6 months of platinum-based chemotherapy.

\section{DNA damage response (DDR) drugs}

PARP (poly (ADP-ribose) polymerase) inhibitors can induce cell death by inhibiting the activity of PARP, which is a protein involved in DNA repair (72). A phase II trial (NCT03958045) is recruiting to combine rucaparib with nivolumab in stage-IV SCLC, evaluating their potential to prolong PFS and OS.

WEE1 is a protein kinase that regulates the cell cycle by serving as a checkpoint preventing DNA replication in the presence of DNA damage (73). The inhibition of WEE1 aims to generate sufficient DNA damage in cancer cells to cause apoptosis and prevent tumor growth. $\mathrm{ZN}-\mathrm{c} 3$ is an orally administered WEE1 inhibitor, which is investigated in phase I/II study (NCT04158336) as monotherapy and in combination with pembrolizumab or talazoparib in solid cancers including SCLC.

Topoisomerase II (Top 2) is an enzyme incredible in DNA replication, chromosome condensation, and chromosome segregation (74). As a result, inhibitors of Top 2 are increasingly utilized in the therapy of various cancers, including lung cancer. Amrubicin, a third-generation anthracycline and potent, which has been permitted for relapsed small cell lung carcinoma in Japan (75). NCT03253068 (phase II) is continuing to explore the overall response rate per RECIST 1.1 as assessed by investigators in subjects with refractory SCLC treated with pembrolizumab combined amrubicin.

PM01183 is a novel antitumor agent binding to selected DNA sequences and promoting apoptosis via double-strand breaks at nanomolar concentrations (76). NCT04253145 is a phase I trial to assess the safety, tolerability, and efficacy of PM01183 combined with atezolizumab in SCLC patients.

\section{Notch pathway inhibitors}

The Notch is a surface receptor in regulating cell proliferation, survival, differentiation, and cell death by interacting with transmembrane ligands on neighboring cells (77), which is one of the most prevalently activated signaling pathways in cancers (78). A study of colorectal carcinoma revealed the blockade of Notch pathway could stimulate tumor-infiltrating $\mathrm{CD}^{+}$cytotoxic $\mathrm{T}$ lymphocytes (CTL), and the production of IFN- $\gamma$, TNF- $\alpha$, IL-1 $\beta$, IL-6, and IL-8 (79). What is more, the PD1 expression on CD8 $8^{+}$ CTL was also declined with the inhabitation of Notch pathway, demonstrating the great potential to assist PD-1 blockers.

Delta-like ligand 3 (DLL3) is an inhibitory Notch ligand that is overexpressed in SCLC cells but hardly expressed in normal tissues (80). AMG 757 is a bispecific antibody targeting DLL3 and CD3, which has been demonstrated to turn T cells to eliminate DLL3 ${ }^{+}$SCLC cells in vitro (80). A phase I trial (NCT03319940) is attempting to compare AMG 757 monotherapy and AMG 757 combined with pembrolizumab in small cell lung carcinoma. Rovalpituzumab tesirine (Rova-T) is an anti-DLL3 conjugate, with early signs of efficacy as a third- or fourth-line setting in SCLC (80). Therefore, NCT03026166 is a phase I/II study of Rova-T with nivolumab \pm ipilimumab for ES-SCLC, where Rova-T with nivolumab and ipilimumab is not appropriate, attributable to dose-limiting toxicities (DLTs). However, Rova- $\mathrm{T}$ plus nivolumab demonstrated some durable responses and required optimizing dose and schedule (81).

\section{Kirsten rat sarcoma viral oncogene bomolog (KRAS) patbway inbibitors}

KRAS activation is one of the most often oncogenic events in some cancers and is correlated with poor survival (82). Although there has not been a clear conclusion in SCLC, several surprising ICIs in KRAS mutant NSCLC have been reported. According to a meta-analysis, ICIs prolonged the OS of KRAS mutant NSCLC, compared with chemotherapy $(\mathrm{P}=0.03)$ (83). Therefore, whether KRAS mutation could also be a potential biomarker for the efficacy of ICIs in SCLC is worth discussion. Plinabulin is a novel agent that inhibits tubulin polymerization and growth of KRAS-driven cancer cells with a manageable safety profile in previous studies (82). Currently, a phase1/2 study (NCT03575793) determines the different outcomes between nivolumab plus plinabulin, and nivolumab 
combined with ipilimumab plus plinabulin in recurrent SCLC patients.

\section{Lysine-specific demethylase 1 (LSD1) inbibitors}

Lysine-specific demethylase 1 (LSD1, KDM1A) promotes cancer cell proliferation, metastasis, and invasion (84). Inhibitors of LSD1 has been validated with significant antitumor activity in vitro and vivo in a series of solid tumors, including SCLC (85). Mounting results in breast mice models showed that the combination of LSD1 inhibitors with PD-1 antibody significantly inhibited tumor growth, decreased Ki-67 level, and enhanced CD8 ${ }^{+}$TILs (86). Overall, these results proved that LSD1 inhibition could be an approach to assist with immunotherapy.

INCB059872 is a demethylase that suppresses LSD1 pathway and being combined with azacytidine and nivolumab in advanced malignancies, including SCLC (NCT02712905, phase I/II). CC-90011 is another demethylase binding to and inhibiting LSD1, which is being tested in combination with nivolumab and EC in ES-SCLC (NCT03850067, phase I/II).

\section{Liver-X nuclear hormone receptor (LXR) agonist}

LXR- $\alpha$ and $\beta$ are vital regulators in lipid and carbohydrate metabolism, cellular differentiation, apoptosis, and many immune responses (87). LXR- $\alpha$ is highly expressed in fat tissue, liver, kidney, intestine, and macrophages, while LXR- $\beta$ expression is universal (88), both of which have demonstrated anticancer activities in a variety of cancer cell lines (89). RGX-104, an oral administered LXR agonist, was proved to upgrade LXR-mediated expression of apolipoprotein E (ApoE), which induced apoptosis of peripheral or tumor-infiltrating MDSCs, and then supported $\mathrm{T}$ cell-engaging antitumor immunity (90). Consequently, this therapy was considered to promote the curative effect of ICIs. Hence, NCT02922764 (phase I) sets up five arms to assess the activity and tolerability of RGX104 monotherapy, RGX-104 plus nivolumab, or ipilimumab or docetaxel and the combination of RGX-104 with pembrolizumab, carboplatin, and pemetrexed.

More details of these above ongoing clinical trials were demonstrated in Table S1.

\section{Future perspectives}

In the next years, ICIs alone or in combination with additional immunotherapeutic agents, targeted therapy, chemotherapy, and (or) radiotherapy promise to reverse the refractory situation of SCLC. Multiple trials are investigating the benefit of ICIs in various neoadjuvant settings. Thus, oncologists have to deal with more difficulty and complicated selectivity.

\section{Development of more predictive biomarkers}

First, lacking potential biomarkers to predict the therapeutic effect of ICIs alone or in combination with other therapies is concerned in SCLC (91). High level of PD-L1 in multiple tumor cells or TME had been assumed as a predictor for a better response and survival after ICIs treatment, which has been approved by the FDA as an indicator for variable solid tumors, including lung cancers. However, recent evidence suggested that the clinical benefit of ICIs might not be restricted in PD-L1 high patients, leading to the full doubt of the predictive capability of PD-L1 (92). Thereby, multimodal detection of PD-L1, such as PD-L1 protein, PD-L1 mRNA, circulating PD-L1, and dynamic PD-L1 monitoring, might provide a comprehensive method for screening candidates for ICIs. Moreover, combined biomarkers, such as PD-L1 combined with tumor mutation burden (TMB) and microsatellite instability-high/mismatch repair deficient, nomograms, and deep learning, could better provide advice for therapeutic decisions (93).

\section{Specific clinical trial designs}

Ten to twenty-five percent of patients beneficial from ICIs are not counted by the traditional response criteria, due to the similar imaging performance of inflammation and tumor growth, the antitumor activity of immunotherapy might be more substantial than measured by standard response criteria (94). Secondly, due to the immunotherapy is based on slow stimulation of the immune system rather than rapidly assaulting tumor cells, the efficacy and antitumor responses are always delayed (95). Thereby, the endpoints of traditional anticancer treatment may be insufficient for immunotherapy, and long-term disease-free survival is more appropriate for immunotherapy (96). Moreover, the assessment of multiple immune cells in the TME or peripheral blood should be counted as additional endpoints for immunotherapy (96).

\section{Enhancement of the efficacy}

Tumor heterogeneity and treatment resistance are the 
most two mechanisms to hinder the efficacy of cancer immunotherapy (92). Liquid biopsies, containing the genomic analysis of circulating free DNA or cancer cells, could noninvasively obtain the level of tumor heterogeneity and also dynamically monitor resistance (97). Moreover, liquid biopsies are promising for personalized medicine to screen optimal combinational therapy (97).

\section{Decreasing the financial toxicity}

Cancer immunotherapies are known as one of the most promising treatments for malignant tumors, while they are also the most expensive ones in cancer, considering the enormous research costs (98). The cost for each lung cancer patient to treat with nivolumab was estimated at $\$ 44,100$ per year (99). Thence, screening potential patients for immunotherapy is critical to reducing the unnecessary financial burden on patients. Meanwhile, cost-effectiveness, cost-benefit, and quality of life (QoL) is another approach to weigh the clinical benefit and financial cost (92).

Understanding the evolving immuno-oncology is increasingly relevant and crucial to solve those problems and define therapeutic strategies and potential target populations of combinational immunotherapy. Ultimately, emerging combinational immunotherapy will transform SCLC into a chronic disease to help patients survive from tumors.

\section{Acknowledgments}

Funding: This study was supported in part by Key Program of Natural Science Research of Anhui Provincial Education Department (KJ2018A0813), a grant from National Natural Science Foundation of China (81802255), Shanghai Pujiang Program (17PJD036) and a grant from Shanghai Municipal Commission of Health and Family Planning Program (20174Y0131), National Key Research \& Development Project (2016YFC0902300), major disease clinical skills enhancement program of 3 year action plan for promoting clinical skills and clinical innovation in municipal hospitals, Shanghai Shen Kang Hospital Development Center Clinical Research Plan of SHDC (16CR1001A), "Dream Tutor" Outstanding Young Talents Program (fkyq1901), Key Disciplines of Shanghai Pulmonary Hospital (2017ZZ02012), grant of Shanghai Science and Technology Commission (16JC1405900).

\section{Footnote}

Peer Review File: Available at http://dx.doi.org/10.21037/jtd-
20-1689

Conflicts of Interest: All authors have completed the ICMJE uniform disclosure form (available at http://dx.doi. org/10.21037/jtd-20-1689). The authors have no conflicts of interest to declare.

Ethical Statement: The authors are accountable for all aspects of the work in ensuring that questions related to the accuracy or integrity of any part of the work are appropriately investigated and resolved.

Open Access Statement: This is an Open Access article distributed in accordance with the Creative Commons Attribution-NonCommercial-NoDerivs 4.0 International License (CC BY-NC-ND 4.0), which permits the noncommercial replication and distribution of the article with the strict proviso that no changes or edits are made and the original work is properly cited (including links to both the formal publication through the relevant DOI and the license). See: https://creativecommons.org/licenses/by-nc-nd/4.0/.

\section{References}

1. Hann CL, Scherpereel A, Hellyer JA, et al. Role of Immunotherapy in Small Cell Lung Cancer, Thymic Epithelial Tumors, and Mesothelioma. Am Soc Clin Oncol Educ Book 2019;39:543-52.

2. Nesbit EG, Leal TA, Kruser TJ. What is the role of radiotherapy for extensive-stage small cell lung cancer in the immunotherapy era? Transl Lung Cancer Res. 2019;8:S153-62.

3. Sharp A, Bhosle J, Abdelraouf F, et al. Development of molecularly targeted agents and immunotherapies in small cell lung cancer. Eur J Cancer2016;60:26-39.

4. Nikanjam M, Patel H, Kurzrock R. Dosing immunotherapy combinations: Analysis of 3,526 patients for toxicity and response patterns. Oncoimmunology 2017;6:e1338997.

5. Lazzari C, Karachaliou N, Bulotta A, et al. Combination of immunotherapy with chemotherapy and radiotherapy in lung cancer: is this the beginning of the end for cancer? Ther Adv Med Oncol 2018;10:1758835918762094.

6. Zou W, Chen L. Inhibitory B7-family molecules in the tumour microenvironment. Nat Rev Immunol 2008;8:467-77.

7. Leung HT, Bradshaw J, Cleaveland JS, et al. Cytotoxic T lymphocyte-associated molecule-4, a high-avidity receptor for CD80 and CD86, contains an intracellular localization 
motif in its cytoplasmic tail. J Biol Chem 1995;270:25107-14.

8. Krummey SM, Ford ML. Braking bad: Novel mechanisms of ctla-4 inhibition of t cell responses. Am J Transplant 2014;14:2685-90.

9. Wing K, Onishi Y, Prieto-Martin P, et al. CTLA-4 control over Foxp3 + regulatory $\mathrm{T}$ cell function. Science 2008;322:271-5.

10. Tang F, Du X, Liu M, et al. Anti-CTLA-4 antibodies in cancer immunotherapy: selective depletion of intratumoral regulatory $\mathrm{T}$ cells or checkpoint blockade? Cell Biosci 2018;8:30.

11. Pai CS, Simons DM, Lu X, et al. Tumor-conditional anti-CTLA4 uncouples antitumor efficacy from immunotherapy-related toxicity. J Clin Invest 2019;129:349-63.

12. Berger JR. PD-1 inhibition: a novel approach to the treatment of progressive multifocal leukoencephalopathy. Ann Transl Med 2019;7:S281.

13. Liu Y, Zugazagoitia J, Ahmed FS, et al. Immune cell PDL1 co-localizes with macrophages and is associated with outcome in PD-1 pathway blockade therapy. Clin Cancer Res 2020;26:970-7.

14. Sponaas A-M, Moharrami NN, Feyzi E, et al. PDL1 expression on plasma and dendritic cells in myeloma bone marrow suggests benefit of targeted anti PD1-PDL1 therapy. PLoS One 2015;10:e0139867.

15. Li A, Barsoumian HB, Schoenhals JE, et al. Indoleamine 2, 3-dioxygenase 1 inhibition targets anti-PD1-resistant lung tumors by blocking myeloid-derived suppressor cells. Cancer Lett 2018;431:54-63.

16. Cheng Y, Li H, Deng Y, et al. Cancer-associated fibroblasts induce PDL1+ neutrophils through the IL6-STAT3 pathway that foster immune suppression in hepatocellular carcinoma. Cell Death Dis 2018;9:422.

17. Lou Y, Diao L, Byers LA, et al. Association of epithelialmesenchymal transition status with PD1/PDL1 expression and a distinct immunophenotype in non-small cell lung cancer: Implications for immunotherapy biomarkers. J Clin Oncol 2014;32:3018.

18. Ahmadzadeh M, Johnson LA, Heemskerk B, et al. Tumor antigen-specific CD8 T cells infiltrating the tumor express high levels of PD-1 and are functionally impaired. Blood. Blood 2009;114:1537-44.

19. Francisco LM, Sage PT, Sharpe AH. The PD 1 pathway in tolerance and autoimmunity. Immunol Rev 2010;236:219-42.

20. Ohaegbulam KC, Assal A, Lazar-Molnar E, et al. Human cancer immunotherapy with antibodies to the PD-1 and PD-L1 pathway. Trends Mol Med 2015;21:24-33.
21. Akinleye A, Rasool Z. Immune checkpoint inhibitors of PD-L1 as cancer therapeutics. J Hematol Oncol 2019;12:92.

22. Horn L, Reck M, Gettinger SN, et al. CheckMate 331: An open-label, randomized phase III trial of nivolumab versus chemotherapy in patients (pts) with relapsed small cell lung cancer (SCLC) after first-line platinum-based chemotherapy (PT-DC). J Clin Oncol 2016;34:TPS8578.

23. Ready N, Farago AF, de Braud F, et al. Third-line nivolumab monotherapy in recurrent SCLC: CheckMate 032. J Thorac Oncol 2019;14:237-44.

24. Goldman JW, Dowlati A, Antonia SJ, et al. Safety and antitumor activity of durvalumab monotherapy in patients with pretreated extensive disease small-cell lung cancer (ED-SCLC). J Clin Oncol 2018;36:8518.

25. Gadgeel SM, Pennell NA, Fidler MJ, et al. Phase II Study of Maintenance Pembrolizumab in Patients with Extensive-Stage Small Cell Lung Cancer (SCLC). J Thorac Oncol 2018;13:1393-9.

26. Sequist LV, Chiang A, Gilbert J, et al. Clinical activity, safety and predictive biomarkers results from a phase Ia atezolizumab (atezo) trial in extensive-stage small cell lung cancer (ES-SCLC). Ann Oncol 2016;27:493-6.

27. Das M, Zhu C, Kuchroo VK. Tim-3 and its role in regulating anti-tumor immunity. Immunol Rev 2017;276:97-111.

28. He Y, Cao J, Zhao C, et al. TIM-3, a promising target for cancer immunotherapy. Onco Targets Ther 2018;11:7005-9.

29. Zhu C, Sakuishi K, Xiao S, et al. An IL-27/NFIL3 signalling axis drives Tim-3 and IL-10 expression and T-cell dysfunction. Nat Commun 2015;6:6072.

30. Zhang Y, Ma CJ, Wang JM, et al. Tim-3 regulates proand anti-inflammatory cytokine expression in human CD14+ monocytes. J Leukoc Biol 2012;91:189-96.

31. Xu LY, Chen DD, He JY, et al. Tim-3 expression by peripheral natural killer cells and natural killer T cells increases in patients with lung cancer--reduction after surgical resection. Asian Pac J Cancer Prev 2014;15:9945-8.

32. Gao X, Zhu Y, Li G, et al. TIM-3 expression characterizes regulatory $\mathrm{T}$ cells in tumor tissues and is associated with lung cancer progression. PLoS One 2012;7:e30676.

33. Xu L, Huang Y, Tan L, et al. Increased Tim-3 expression in peripheral NK cells predicts a poorer prognosis and Tim-3 blockade improves NK cell-mediated cytotoxicity in human lung adenocarcinoma. Int Immunopharmacol 2015;29:635-41.

34. Long L, Zhang X, Chen F, et al. The promising immune checkpoint LAG-3: from tumor microenvironment to 
cancer immunotherapy. Genes Cancer 2018;9:176-89.

35. Buisson S, Triebel F. LAG-3 (CD223) reduces macrophage and dendritic cell differentiation from monocyte precursors. Immunology 2005;114:369-74.

36. Antonia S, Goldberg SB, Balmanoukian A, et al. Safety and antitumour activity of durvalumab plus tremelimumab in non-small cell lung cancer: a multicentre, phase $1 \mathrm{~b}$ study. Lancet Oncol 2016;17:299-308.

37. Antonia SJ, López-Martin JA, Bendell J, et al. Nivolumab alone and nivolumab plus ipilimumab in recurrent smallcell lung cancer (CheckMate 032): a multicentre, openlabel, phase 1/2 trial. Lancet Oncol 2016;17:883-95.

38. Ready N, Owonikoko TK, Postmus PE, et al. CheckMate 451: A randomized, double-blind, phase III trial of nivolumab (nivo), nivo plus ipilimumab (ipi), or placebo as maintenance therapy in patients (pts) with extensive-stage disease small cell lung cancer (ED-SCLC) after first-line platinum-based doublet chemotherapy (PT-DC). J Clin Oncol 2016;34:TPS8579.

39. Waldmann TA. Cytokines in Cancer Immunotherapy. Cold Spring Harb Perspect Biol 2018;10:a028472.

40. Wrangle JM, Velcheti V, Patel MR, et al. ALT-803, an IL-15 superagonist, in combination with nivolumab in patients with metastatic non-small cell lung cancer: a nonrandomised, open-label, phase $1 \mathrm{~b}$ trial. Lancet Oncol 2018;19:694-704.

41. Dajon M, Iribarren K, Cremer I. Dual roles of TLR7 in the lung cancer microenvironment. Oncoimmunology 2015;4:e991615.

42. Ponath P, Menezes D, Pan C, et al. A Novel, Fully Human Anti-fucosyl-GM1 Antibody Demonstrates Potent In Vitro and In Vivo Antitumor Activity in Preclinical Models of Small Cell Lung Cancer. Clin Cancer Res 2018;24:5178-89.

43. Loeb DM, Sukumar S. The role of WT1 in oncogenesis: tumor suppressor or oncogene? Int J Hematol 2002;76:117-26.

44. Xu C, Wu C, Xia Y, et al. WT1 promotes cell proliferation in non-small cell lung cancer cell lines through upregulating cyclin D1 and p-pRb in vitro and in vivo. PLoS One 2013;8:e68837.

45. Bjordahl R, Mahmood S, Gaidarova S, et al. Abstract 3576: FT500, an off-the-shelf NK cell cancer immunotherapy derived from a master pluripotent cell line, enhances T-cell activation and recruitment to overcome checkpoint blockade resistance. Cancer Res 2018;78:3576.

46. Chiappori AA, Soliman H, Janssen WE, et al. INGN-225: a dendritic cell-based p53 vaccine (Ad.p53-DC) in small cell lung cancer: observed association between immune response and enhanced chemotherapy effect. Expert Opin Biol Ther 2010;10:983-91.

47. Arriola E, Wheater M, Galea I, et al. Outcome and Biomarker Analysis from a Multicenter Phase 2 Study of Ipilimumab in Combination with Carboplatin and Etoposide as First-Line Therapy for Extensive-Stage SCLC. J Thorac Oncol 2016;11:1511-21.

48. Reck M, Luft A, Szczesna A, et al. Phase III Randomized Trial of Ipilimumab Plus Etoposide and Platinum Versus Placebo Plus Etoposide and Platinum in Extensive-Stage Small-Cell Lung Cancer. J Clin Oncol 2016;34:3740-8.

49. Kim Y, Keam B, Ock C-Y, et al. A phase II study of pembrolizumab and paclitaxel in refractory extensive disease small cell lung cancer. J Clin Oncol 2018;36:8575.

50. Weiss GJ, Waypa J, Blaydorn L, et al. A phase Ib study of pembrolizumab plus chemotherapy in patients with advanced cancer (PembroPlus). Br J Cancer 2017;117:33-40.

51. Rudin CM, Awad MM, Navarro A, et al. KEYNOTE-604: Pembrolizumab (pembro) or placebo plus etoposide and platinum (EP) as first-line therapy for extensive-stage (ES) small-cell lung cancer (SCLC). J Clin Oncol 2020;38:9001.

52. Horn L, Mansfield AS, Szczęsna A, et al. First-Line Atezolizumab plus Chemotherapy in Extensive-Stage Small-Cell Lung Cancer. N Engl J Med 2018;379:2220-9.

53. Rizvi NA, Hellmann MD, Brahmer JR, et al. Nivolumab in Combination With Platinum-Based Doublet Chemotherapy for First-Line Treatment of Advanced Non-Small-Cell Lung Cancer. J Clin Oncol 2016;34:2969-79.

54. Paz-Ares L, Dvorkin M, Chen Y, et al. Durvalumab plus platinum-etoposide versus platinum-etoposide in firstline treatment of extensive-stage small-cell lung cancer (CASPIAN): a randomised, controlled, open-label, phase 3 trial. Lancet 2019;394:1929-39.

55. Krebs M, Ross K, Kim S, et al. P1.15-004 An OpenLabel, Multitumor Phase II Basket Study of Olaparib and Durvalumab (MEDIOLA): Results in Patients with Relapsed SCLC. J Thorac Oncol 2017;12:S2044-5.

56. Brabander T, van der Zwan WA, Teunissen JJM, et al. Long-Term Efficacy, Survival, and Safety of [(177) Lu-DOTA(0), $\operatorname{Tyr}(3)$ ]octreotate in Patients with Gastroenteropancreatic and Bronchial Neuroendocrine Tumors. Clin Cancer Res 2017;23:4617-24.

57. Kim C, Subramaniam D, Liu S, et al. P2.12-03 Phase I/II Trial Of 177Lu-DOTA0-Tyr3-Octreotate (Lutathera) And Nivolumab for Patients with Extensive-Stage Small Cell Lung Cancer (ES-SCLC). J Thorac Oncol 2018;13:S791.

58. Owonikoko TK, Higgins KA, Chen Z, et al. A randomized phase II study of tremelimumab and durvalumab with or 
without radiation for patients with relapsed small cell lung cancer (SCLC). J Clin Oncol 2019;37:8515.

59. Perez BA, Kim S, Dilling TJ, et al. A Prospective Single Arm Phase I/II Study: Consolidative Ipilimumab and Nivolumab with Thoracic Radiotherapy after Platinum Based Chemotherapy for Patients with Extensive-Stage Small Cell Lung Cancer. Int J Radiat Oncol Biol Phys 2019;105:S36.

60. Schmittnaegel M, Rigamonti N, Kadioglu E, et al. Dual angiopoietin-2 and VEGFA inhibition elicits antitumor immunity that is enhanced by PD-1 checkpoint blockade. Sci Transl Med 2017;9:eaak9670.

61. Allen E, Jabouille A, Rivera LB, et al. Combined antiangiogenic and anti-PD-L1 therapy stimulates tumor immunity through HEV formation. Sci Transl Med 2017;9:eaak9679.

62. Shen G, Zheng F, Ren D, et al. Anlotinib: a novel multitargeting tyrosine kinase inhibitor in clinical development. J Hematol Oncol 2018;11:120.

63. Ferguson FM, Gray NS. Kinase inhibitors: the road ahead. Nat Rev Drug Discov 2018;17:353.

64. Schöffski P, Gordon M, Smith DC, et al. Phase II randomised discontinuation trial of cabozantinib in patients with advanced solid tumours. Eur J Cancer 2017;86:296-304.

65. Kazazi-Hyseni F, Beijnen JH, Schellens JHM. Bevacizumab. Oncologist 2010;15:819-25.

66. Pacheco J, Schenk E. CDK4/6 inhibition alone and in combination for non-small cell lung cancer. Oncotarget 2019;10:618-9.

67. Schettini F, De Santo I, Rea CG, et al. CDK 4/6 Inhibitors as Single Agent in Advanced Solid Tumors. Front Oncol 2018;8:608.

68. Zhang H, Christensen CL, Dries R, et al. CDK7 Inhibition Potentiates Genome Instability Triggering Anti-tumor Immunity in Small Cell Lung Cancer. Cancer Cell 2020;37:37-54.e9.

69. Chang C-I, Liao JC, Kuo L. Macrophage arginase promotes tumor cell growth and suppresses nitric oxidemediated tumor cytotoxicity. Cancer Res 2001;61:1100-6.

70. Shatanawi A, Qasrawi H. Arginase Inhibition Suppresses Breast Cancer Cell Proliferation. FASEB J 2017;31:lb529.

71. Agnello G, Alters SE, Rowlinson SW. Preclinical safety and antitumor activity of the arginine-degrading therapeutic enzyme pegzilarginase, a PEGylated, cobaltsubstituted recombinant human arginase 1 . Transl Res 2020;217:11-22.

72. Sachdev E, Tabatabai R, Roy V, et al. PARP Inhibition in Cancer: An Update on Clinical Development. Target Oncol 2019;14:657-79.

73. Do K, Doroshow JH, Kummar S. Wee1 kinase as a target for cancer therapy. Cell Cycle 2013;12:3159-64.

74. Hande KR. Topoisomerase II inhibitors. Update Cancer Ther 2008;3:13-26.

75. Horita N, Yamamoto M, Sato T, et al. Amrubicin for relapsed small-cell lung cancer: a systematic review and meta-analysis of 803 patients. Sci Rep 2016;6:18999.

76. Leal JFM, Martínez-Díez M, García-Hernández V, et al. PM01183, a new DNA minor groove covalent binder with potent in vitro and in vivo anti-tumour activity. $\mathrm{Br} \mathrm{J}$ Pharmacol 2010;161:1099-110.

77. Kopan R. Notch signaling. Cold Spring Harb Perspect Biol 2012;4:a011213.

78. Yuan $\mathrm{X}, \mathrm{Wu} \mathrm{H}, \mathrm{Xu} \mathrm{H}$, et al. Notch signaling: An emerging therapeutic target for cancer treatment. Cancer Lett 2015;369:20-7.

79. Yu W, Wang Y, Guo P. Notch signaling pathway dampens tumor-infiltrating CD8(+) T cells activity in patients with colorectal carcinoma. Biomed Pharmacother 2018;97:535-42.

80. Owen DH, Giffin MJ, Bailis JM, et al. DLL3: an emerging target in small cell lung cancer. J Hematol Oncol 2019;12:61.

81. Malhotra J, Nikolinakos P, Leal T, et al. Ph1/2 study of Rova-T in combination with nivolumab (Nivo) \pm ipilimumab (Ipi) for patients (pts) with $2 \mathrm{~L}+$ extensive-stage (ED) SCLC. J Clin Oncol 2019;37:8516.

82. Cimino PJ, Huang L, Du L, et al. Plinabulin, an inhibitor of tubulin polymerization, targets KRAS signaling through disruption of endosomal recycling. Biomed Rep 2019;10:218-24.

83. Kim JH, Kim HS, Kim BJ. Prognostic value of KRAS mutation in advanced non-small-cell lung cancer treated with immune checkpoint inhibitors: A meta-analysis and review. Oncotarget 2017;8:48248-52.

84. Yang GJ, Lei PM, Wong SY, et al. Pharmacological Inhibition of LSD1 for Cancer Treatment. Molecules 2018;23:3194.

85. Stewart CA, Byers LA. Altering the Course of Small Cell Lung Cancer: Targeting Cancer Stem Cells via LSD1 Inhibition. Cancer cell 2015;28:4-6.

86. Qin Y, Vasilatos SN, Chen L, et al. Inhibition of histone lysine-specific demethylase 1 elicits breast tumor immunity and enhances antitumor efficacy of immune checkpoint blockade. Oncogene 2019;38:390-405.

87. Patel MB, Oza NA, Anand IS, et al. Liver x receptor: a novel therapeutic target. Indian J Pharm Sci 2008;70:135-44. 
88. Bischoff ED, Daige CL, Petrowski M, et al. Nonredundant roles for LXRalpha and LXRbeta in atherosclerosis susceptibility in low density lipoprotein receptor knockout mice. J Lipid Res 2010;51:900-6.

89. Liu S, Cao H, Chen D, et al. LXR ligands induce apoptosis of EGFR-TKI-resistant human lung cancer cells in vitro by inhibiting Akt-NF- $\kappa \mathrm{B}$ activation. Oncol Lett 2018;15:7168-74.

90. Tavazoie MF, Pollack I, Tanqueco R, et al. LXR/ApoE Activation Restricts Innate Immune Suppression in Cancer. Cell 2018;172:825-40.e18.

91. Mahoney KM, Rennert PD, Freeman GJ. Combination cancer immunotherapy and new immunomodulatory targets. Nat Rev Drug Discov 2015;14:561-84.

92. Ventola CL. Cancer Immunotherapy, Part 3: Challenges and Future Trends. P T 2017;42:514-21.

93. Yang Q, Xu Z, Zheng L, et al. Multimodal detection of PD-L1: reasonable biomarkers for immune checkpoint inhibitor. Am J Cancer Res 2018;8:1689-96.

Cite this article as: Guo H, He Y, Chen P, Wang L, Li W, Chen B, Liu Y, Wang H, Zhao S, Zhou C. Combinational immunotherapy based on immune checkpoints inhibitors in small cell lung cancer: is this the beginning to reverse the refractory situation? J Thorac Dis 2020;12(10):6070-6089. doi: 10.21037/jtd20-1689
94. Wolchok JD, Hoos A, O'Day S, et al. Guidelines for the evaluation of immune therapy activity in solid tumors: immune-related response criteria. Clin Cancer Res 2009;15:7412-20.

95. Hoos A, Eggermont AMM, Janetzki S, et al. Improved endpoints for cancer immunotherapy trials. J Natl Cancer Inst 2010;102:1388-97.

96. Alatrash G, Jakher H, Stafford PD, et al. Cancer immunotherapies, their safety and toxicity. Expert Opin Drug Saf 2013;12:631-45.

97. Xu C, Offin M, Paik PK, Li BT. Liquid biopsy guided precision therapy for lung cancers. J Thorac Dis 2018;10:S4173-5.

98. Kottschade LA. The Future of Immunotherapy in the Treatment of Cancer. Semin Oncol Nurs 2019;35:150934.

99. Tartari F, Santoni M, Burattini L, et al. Economic sustainability of anti-PD-1 agents nivolumab and pembrolizumab in cancer patients: Recent insights and future challenges. Cancer Treat Rev 2016;48:20-4. 
Supplementary

\begin{tabular}{|c|c|c|c|c|c|c|c|c|}
\hline ICls & Other therapies & Target & Clinical trial & Phase & Stage & Status & Estimated Study Completion & Primary outcome measures \\
\hline \multicolumn{9}{|l|}{ Anti-angiogenic agents } \\
\hline Nivolumab & Anlotinib (AL3818) & VEGFR, FGFR, PDGFR, c-kit & NCT04165330 & Phase $1 / I$ & Unknown & Recruiting & 31-Dec-22 & ORR \\
\hline Sintilimab & Anlotinib (AL3818) & VEGFR, FGFR, PDGFR, c-kit & NCT04055792 & Phase II & Unknown & Recruiting & 1-Mar-22 & PFS \\
\hline Nivolumab & Vorolanib (X-82) & VEGFR/PDGFR & NCT03583086 & Phase I/II & Stage IV & Recruiting & Jul-24 & RP2D, Phase II best response \\
\hline Nivolumab + Ipilimumab & XL184 (cabozantinib) & MET, VEGFR 2, AXL, ROS1, RET & NCT04079712 & Phase II & Unknown & Not yet recruiting & 17-Jul-22 & Overall response rate \\
\hline Durvalumab & Bevacizumab & VEGF-A & NCT02734004 & Phase $1 / I$ & Unknown & Recruiting & 5-Aug-22 & DCR, ORR \\
\hline \multicolumn{9}{|c|}{ Cyclin-dependent protein kinase inhibitors } \\
\hline \multicolumn{9}{|l|}{ Arginase and its inhibitor } \\
\hline Pembrolizumab & Pegzilarginase & Arginase 1 & NCT03371979 & Phase I/II & Unknown & Active, not recruiting & Dec-20 & TEAEs, ORR \\
\hline Pembrolizumab + Epacadostat & INCB001158 & Arginase & NCT03361228 & Phase I/II & Unknown & Completed & 30-Mar-19 & TEAEs, ORR \\
\hline \multicolumn{9}{|l|}{ DNA damage response drugs } \\
\hline Nivolumab & Rucaparib & PARP & NCT03958045 & Phase II & Stage IV & Recruiting & Jul-24 & PFS \\
\hline Pembrolizumab & Amrubicin & Top 2 & NCT03253068 & Phase II & ES & Recruiting & 31-Aug-20 & Overall response rate \\
\hline Pembrolizumab & $\mathrm{ZN}-\mathrm{C3}$ & WEE1 & NCT04158336 & Phase $1 / I$ & Unknown & Recruiting & Aug-23 & MTD, RP2D, ORR \\
\hline Atezolizumab & PM01183 & Trans-activated transcription & NCT04253145 & Phase I & Unknown & Recruiting & Feb-22 & MTD, RD \\
\hline \multicolumn{9}{|l|}{ Notch pathway inhibitors } \\
\hline Pembrolizumab & AMG-757 & DLL3 & NCT03319940 & Phase I & Unknown & Recruiting & 4-Aug-23 & DLTs, TEAEs, significant changes \\
\hline Nivolumab + Ipilimumab & Rovalpituzumab tesirine & DLL3 & NCT03026166 & Phase $1 / I$ & Unknown & Completed & 3-Jul-19 & CBR, DOR, ORR, PFS, OS \\
\hline \multicolumn{9}{|l|}{ KRAS pathway inhibitors } \\
\hline Nivolumab + Ipilimumab & Plinabulin & Tubulin polymerization & NCT03575793 & Phase $1 / I$ & ES & Recruiting & Sep-22 & MTD, PFS \\
\hline \multicolumn{9}{|l|}{ LSD1 inhibitors } \\
\hline Nivolumab & CC-90011 & LSD1 & NCT03850067 & Phase I/II & ES & Recruiting & 30-Sep-22 & DLT, MTD, AEs \\
\hline Nivolumab & INCB059872 & LSD1 & NCT02712905 & Phase I/II & Unknown & Recruiting & 14-Dec-21 & AEs, RD \\
\hline \multicolumn{9}{|l|}{ LXR inhibitors } \\
\hline Docetaxel & RGX-104 & LXR & NCT02922764 & Phase I & Unknown & Recruiting & Mar-21 & MTD, Overall response rate, PFS, TEAEs \\
\hline
\end{tabular}

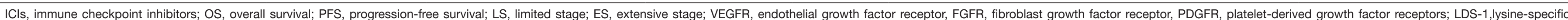

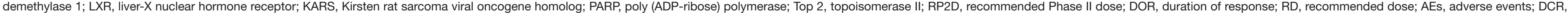
disease control rate; DLTs, dose-limiting toxicities; SAEs, serious adverse events; MTD, maximum tolerated dose; ORR, objective response rate; RR, response rate; TEAEs, treatment-emergent adverse events; CBR, clinical benefit rate. 